\title{
Influence of Mg Doping on the Morphological, Optical, and Structural Properties of InGaN/GaN Multiple Quantum Wells
}

\author{
Z. CHEN,,${ }^{1,2,3}$ N. FICHTENBAUM, ${ }^{1}$ D. BROWN,${ }^{1}$ S. KELLER, ${ }^{1}$ \\ U.K. MISHRA, ${ }^{1}$ S.P. DENBAARS, ${ }^{2}$ and S. NAKAMURA ${ }^{2}$ \\ 1.-Electrical and Computer Engineering Department, University of California, Santa Barbara, \\ CA 93106, USA. 2.-Materials Department, University of California, Santa Barbara, CA 93106, \\ USA. 3.-e-mail: zhen@ece.ucsb.edu
}

\begin{abstract}
In this report, the influence of magnesium doping on the characteristics of InGaN/GaN multiple quantum wells (MQWs) was investigated by means of atomic force microscopy (AFM), photoluminescence (PL), and X-ray diffraction (XRD). Five-period InGaN/GaN MQWs with different magnesium doping levels were grown by metalorganic chemical vapor deposition. The AFM measurements indicated that magnesium doping led to a smoother surface morphology. The V-defect density was observed to decrease with increasing magnesium doping concentration from $\sim 10^{9} \mathrm{~cm}^{-2}$ (no doping) to $\sim 10^{6} \mathrm{~cm}^{-2}$ $\left(\mathrm{Cp}_{2} \mathrm{Mg}: 0.04 \mathrm{sccm}\right)$ and further to $0\left(\mathrm{Cp}_{2} \mathrm{Mg}: 0.2 \mathrm{sccm}\right)$. The PL measurements showed that magnesium doping resulted in stronger emission, which can be attributed to the screening of the polarization-induced band bending. $\mathrm{XRD}$ revealed that magnesium doping had no measurable effect on the indium composition and growth rate of the MQWs. These results suggest that magnesium doping in MQWs might improve the optical properties of GaN photonic devices.
\end{abstract}

Key words: InGaN, multiple quantum wells (MQWs), V-defects, metalorganic chemical vapor deposition (MOCVD), photoluminescence (PL), atomic force microscopy (AFM)

\section{INTRODUCTION}

A high density of V-defects $\left(\sim 10^{9} \mathrm{~cm}^{-2}\right)$ is widely observed in InGaN single layers and multiple quantum wells (MQWs). ${ }^{1-3} \mathrm{~A}$ transmission electron microscopy (TEM) investigation has shown that Vdefects can penetrate the MQW structure, thus degrading the properties of the MQWs. ${ }^{4}$ A near-field scanning optical microscopy study showed that strong fluctuations in the quantum well photoluminescence as well as a tenfold enhancement of deep-level-related emission at lower energies occurred at pits in the heterostructure. ${ }^{5}$ Moreover, it was reported that there is an essential link between

(Received August 2, 2007; accepted October 22, 2007;

published online December 6, 2007)
V-defects and the mechanism of current transport through the GaN light-emitting diode (LED) $p-n$ junction. ${ }^{6,7}$ According to these reports, tunneling behavior dominated throughout all injection regimes in a device with a high density of V-defects in the space-charge region. These V-defects acted as deep-level carrier traps, resulting in a high leakage current. ${ }^{6,7}$ It is therefore desirable to suppress the generation of the V-defects.

One study showed that V-defects contained a threading dislocation at the bottom. ${ }^{8}$ Another report showed that V-defects can be generated at stacking mismatch boundaries induced by stacking faults. These stacking faults are formed within MQWs due to the strain relaxation, especially in MQWs with a high indium composition. ${ }^{9}$ Liliental-Weber 
suggested that rough surface areas such as islandisland coalescence fronts, the areas surrounding the intersection of a dislocation with the growth surface, and impurity clusters are all places where Vdefects can originate. ${ }^{10}$ Northrup and Neugebauer performed calculations for a number of low-index indium-terminated GaN surfaces and showed that indium behaves as a differential surfactant, reducing the energy of the (1011) surface relative to the (0001) surface. ${ }^{11}$ This effect promotes the formation of large (1011)-faceted dislocation pits on the (0001) surface of InGaN. Therefore, V-defects are difficult to avoid in InGaN MQWs when there is a large mismatch strain between the epilayers and substrates. To our knowledge, there are no prior reports about the growth of V-defect-free InGaN MQWs.

In this letter, we report on Mg-doped InGaN MQWs grown by MOCVD and their optical properties, which were studied by photoluminescence (PL) as a function of the $\mathrm{Mg}$ doping level. Our experimental results show that $\mathrm{Mg}$ doping can decrease the density of V-defects dramatically and even suppress the formation of V-defects completely due to its ability to enhance lateral growth. Our AFM measurements indicate that $\mathrm{Mg}$ doping induced two-dimensional growth of the InGaN. To our knowledge, there is no prior report on the growth of Mg-doped InGaN/GaN MQWs and their optical properties.

\section{EXPERIMENTAL}

InGaN/GaN MQWs were grown on (0001) sapphire substrates using MOCVD. A set of samples with varying $\mathrm{Mg}$ doping concentrations were grown to study the effect of $\mathrm{Mg}$ in the InGaN quantum well. For each epitaxial film, a thin GaN buffer layer with a thickness of about $25 \mathrm{~nm}$ was grown at $650^{\circ} \mathrm{C}$ on the sapphire substrate. The substrate temperature was increased to $1150^{\circ} \mathrm{C}$ to grow a GaN film with a thickness of about $2 \mu \mathrm{m}$. Then, a five-period InGaN/GaN MQWs was grown on the thick GaN layer at $800^{\circ} \mathrm{C}$. Trimethylgallium (TMGa), trimethylindium (TMIn), and ammonia $\left(\mathrm{NH}_{3}\right)$ were used as $\mathrm{Ga}, \mathrm{In}$, and $\mathrm{N}$ sources, respectively. The well width and the barrier width were about $3 \mathrm{~nm}$ and $10 \mathrm{~nm}$, respectively. The $\mathrm{Cp}_{2} \mathrm{Mg}$ doping precursor flow was varied from $0 \mathrm{sccm}$ to $0.04 \mathrm{sccm}$ and $0.2 \mathrm{sccm}$ during InGaN well growth for the different samples studied.

\section{RESULTS AND DISCUSSIONS}

Figure 1a shows the undoped InGaN MQW sample with the typically observed surface morphology in a $5 \mu \mathrm{m} \times 5 \mu \mathrm{m}$ AFM scan. Regions with V-defect densities as high as $1 \times 10^{9} \mathrm{~cm}^{-2}$ were observed in this sample. The root-mean-square (RMS) roughness was $1.35 \mathrm{~nm}$ for the scan area. The V-defect density was reduced to $4 \times 10^{6} \mathrm{~cm}^{-2}$ using a $\mathrm{Cp}_{2} \mathrm{Mg}$ flow of $0.04 \mathrm{sccm}$, as shown in Fig. 1b. With the increase of the $\mathrm{Cp}_{2} \mathrm{Mg}$ flow from $0.04 \mathrm{sccm}$ to
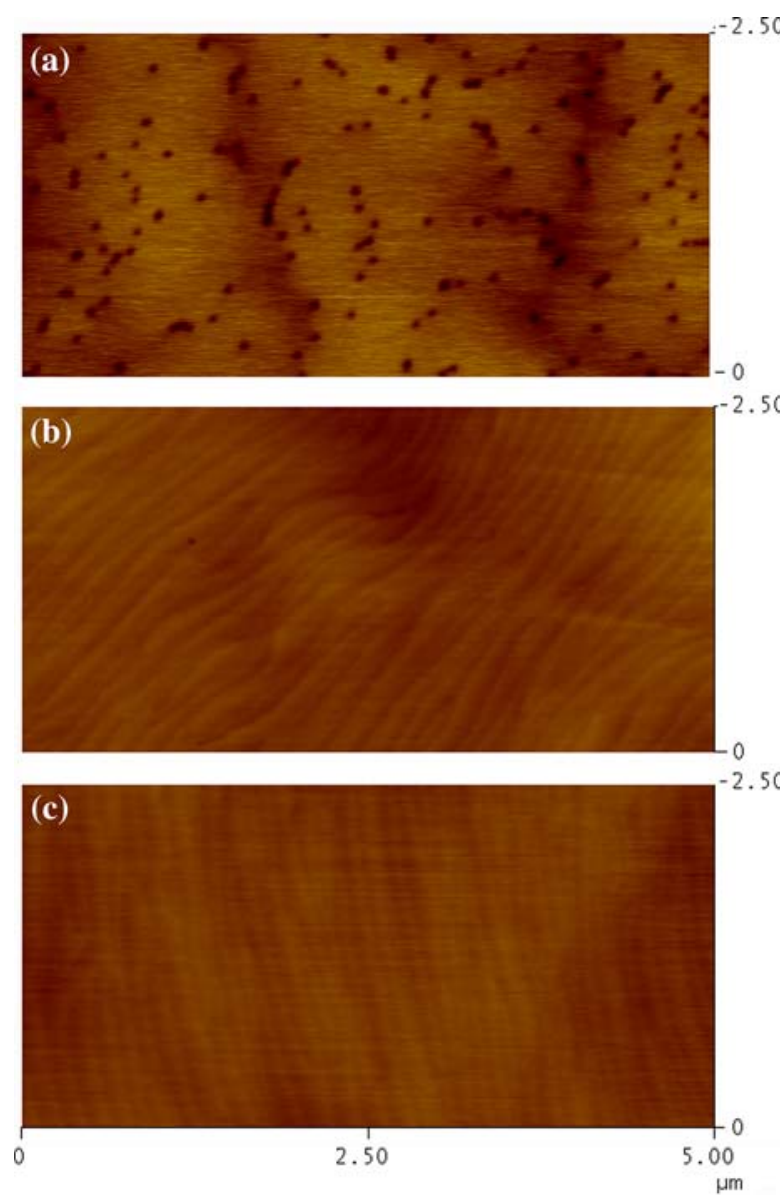

Fig. 1. $5 \mu \mathrm{m} \times 5 \mu \mathrm{m}$ AFM images of InGaN/GaN MQWs at different Mg doping levels: (a) $0 \mathrm{sccm}$, (b) $0.04 \mathrm{sccm}$, and (c) $0.2 \mathrm{sccm}$. The height scale is $10 \mathrm{~nm}$ for all samples.

$0.2 \mathrm{sccm}$, the V-defects disappeared completely, as shown in Fig. 1c. The surfaces became smoother with $\mathrm{Mg}$ doping. The details of the $\mathrm{V}$-defect structures can be seen in the $1 \times 1 \mu \mathrm{m}$ AFM scans, shown in Fig. 2. Figure 2a-c shows AFM images of the same Mg-doped InGaN MQWs samples with $\mathrm{Cp}_{2} \mathrm{Mg}$ flows of $0 \mathrm{sccm}, 0.04 \mathrm{sccm}$, and $0.2 \mathrm{sccm}$, respectively. The surfaces of the Mg-doped InGaN MQWs are characterized by nearly equally spaced and parallel steps, with heights of about one $\mathrm{GaN}$ monolayer, implying a step flow growth mode. $\mathrm{Mg}$ doping enhanced two-dimensional growth significantly.

We attributed the reduction in V-defect density to the $\mathrm{Mg}$ doping. It was reported that introducing $\mathrm{Mg}$ in the GaN lateral epitaxial overgrowth (LEO) process promoted faster coalescence. ${ }^{12}$ It was also found that doping $\mathrm{AlGaN}$ films with $\mathrm{Mg}$ assisted in the coalescence of AlGaN LEO ${ }^{13}$ Beaumont et al. investigated Mg-induced changes in the selective growth of GaN. ${ }^{14}$ They showed that $\mathrm{Mg}$ incorporation favored the adsorption of $\mathrm{Ga}$ on the (1011) facets, but conversely impeded its absorption on the (0001) facets. The V-defects are open hexagonal, 

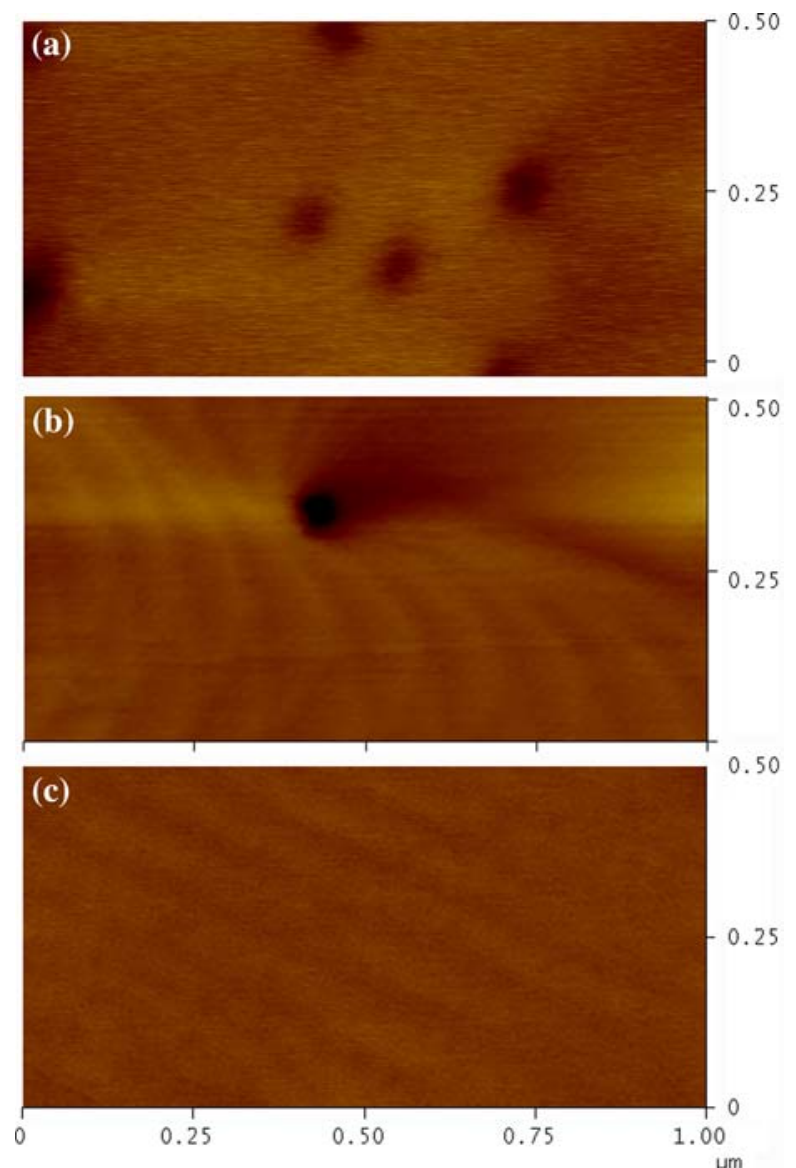

Fig. 2. $1 \mu \mathrm{m} \times 1 \mu \mathrm{m}$ AFM images of InGaN/GaN MQWs at different Mg doping levels: (a) $0 \mathrm{sccm}$, (b) $0.04 \mathrm{sccm}$, and (c) $0.2 \mathrm{sccm}$. The height scale is $10 \mathrm{~nm}$ for all samples.

inverted pyramids with $(10 \overline{1} 1)$ sidewalls, which are inclined about $30^{\circ}$ relative to the $c$-axis. In our case, introducing $\mathrm{Mg}$ improved $\mathrm{Ga}$ incorporation on the (1011) V-defect sidewalls and increased the growth rate of $\mathrm{GaN}$ on these facets. Therefore, $\mathrm{Mg}$ doping reduced the $\mathrm{V}$-defects dramatically and resulted in a $2 \mathrm{D}$ growth mode, which can be seen in the AFM scans.

PL spectra were measured at room temperature (RT) using a $325 \mathrm{~nm}$ KIMMON He-Cd laser source. The RT PL spectra of the Mg doping series are shown in Fig. 3 for comparison. The PL emission intensity of the Mg-doped InGaN MQW sample with a $\mathrm{Cp}_{2} \mathrm{Mg}$ flow of $0.04 \mathrm{sccm}$ was about six times higher than that of the undoped InGaN MQW sample. No deep-level- or Mg-related luminescence was found in the Mg-doped sample. Moreover, the Mg-doped sample had a $15 \mathrm{~nm}$ blue shift compared to the undoped InGaN MQW sample.

These phenomena can be explained as follows. It is well know that a large polarization field exists in (0001) InGaN MQWs. Due to the quantum confined Stark effect (QCSE), the energy band of the MQW is tilted by the polarization field, resulting in a smaller transition energy of the QWs. When the samples

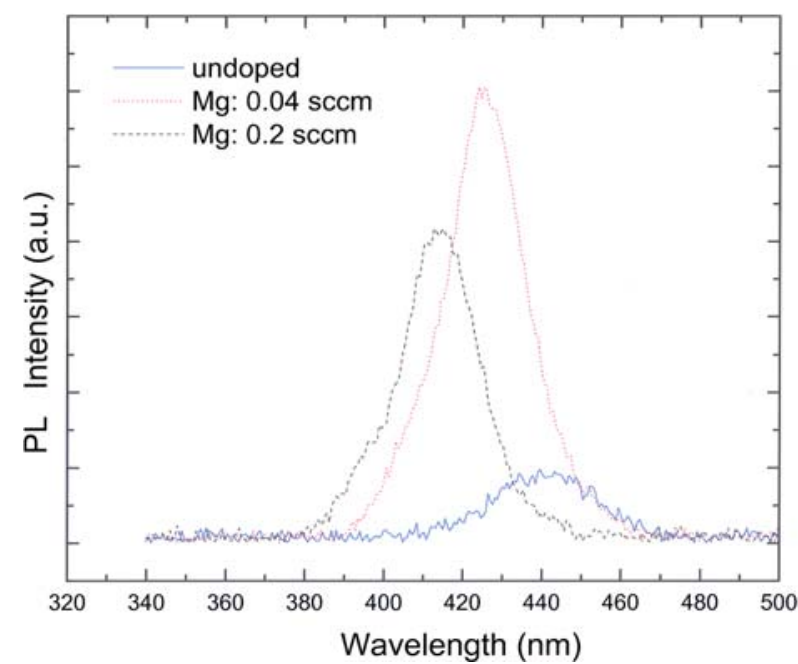

Fig. 3. PL spectra of InGaN/GaN MQWs at different Mg doping levels.

were doped with $\mathrm{Mg}$, some of the $\mathrm{Mg}$ acceptors were ionized and generated holes. These carriers screened the polarization field, weakening the QCSE, and increasing the transition energy (i.e. a blue shift). The emission intensity was higher due to the increased overlap between the hole and electron populations. Heavy $\mathrm{Mg}$ doping is known to cause weak Mg-related deep-level emission in group III nitrides. Light magnesium doping in the well contributed to the high emission in this study. Increasing the $\mathrm{Cp}_{2} \mathrm{Mg}$ flow rate to $0.2 \mathrm{sccm}$ further blue-shifted the emission peak but decreased the emission intensity due to competition with the $\mathrm{Mg}$ related donor-acceptor emission.

The InGaN MQW samples were also characterized using a Philips double-crystal high-resolution X-ray diffraction (HRXRD) system using $\mathrm{Cu} \mathrm{K} \alpha_{1}$ radiation. The $\omega-2 \theta$ scan curve showed higher-order satellite peaks, indicating a good $\operatorname{In}_{x} \mathrm{Ga}_{1-x} \mathrm{~N} / \mathrm{GaN}$ interface. XRD revealed that magnesium doping had no measurable effect on the indium composition and growth rate of the MQWs.

\section{CONCLUSIONS}

We studied the influence of $\mathrm{Mg}$ doping on the morphological, optical, and structural properties of InGaN/GaN MQWs grown on sapphire by MOCVD. Our PL measurements showed that Mg doping resulted in stronger emission and peak blue shift. These effects were attributed to screening of the band bending caused by piezoelectric and spontaneous polarization fields. The AFM measurements indicated that $\mathrm{Mg}$ doping improved the surface morphology of the MQWs and enhanced twodimensional growth. The density of the V-defects was observed to decrease from $\sim 10^{9} \mathrm{~cm}^{-2}$ (undoped) to $\sim 10^{6} \mathrm{~cm}^{-2}$ and further to zero with increasing $\mathrm{Mg}$ doping concentration. 


\section{OPEN ACCESS}

This article is distributed under the terms of the Creative Commons Attribution Noncommercial License which permits any noncommercial use, distribution, and reproduction in any medium, provided the original author(s) and source are credited.

\section{REFERENCES}

1. Y. Chen, T. Takeuchi, H. Amano, I. Akasaki, N. Yamada, Y. Kaneko, and S.Y. Wang, Appl. Phys. Lett. 72, 710 (1998).

2. P. Vennegues, B. Beaumont, M. Vaille, and P. Gibart, Appl. Phys. Lett. 70, 2434 (1997).

3. X.H. Wu, C.R. Elsass, A. Abare, M. Mack, S. Keller, P.M. Petroff, S.P. DenBaars, and J.S. Speck, Appl. Phys. Lett. 72 692 (1998).

4. D. Cherns, S.J. Henley, and F.A. Ponce, Appl. Phys. Lett. 78, 2691 (2001).

5. P.A. Crowell, D.K. Young, S. Keller, E.L. Hu, and D.D. Awschalom, Appl. Phys. Lett. 72, 927 (1998).
6. X.A. Cao, E.B. Stokes, P.M. Sandvik, S.F. LeBoeuf, J. Kretchmer, and D. Walker, IEEE Electron. Dev. Lett. 23, 535 (2002).

7. X.A. Cao, E.B. Stokes, S.F. LeBoeuf, P.M. Sandvik, J. Kretchmer, and D. Walker, Mat. Res. Soc. Symp. Proc. $722, \mathrm{~K} 231$ (2002).

8. N. Sharma, P. Thomas, D. Tricker, and C. Humphreys, Appl. Phys. Lett. 77, 1274 (2000).

9. H.K. Cho, J.Y. Lee, G.M. Yang, and C.S. Kim, Appl. Phys. Lett. 79, 215 (2001).

10. Z. Lillental-Weber, Y. Chen, S. Ruvimov, and J. Washburn, Phys. Rev. Lett. 79, 2835 (1997).

11. J.E. Northrup and J. Neugebauer, Phys. Rev. B 60, R8473 (1999).

12. S. Haffouz, B. Beaumont, and P. Gibart, MRS Internet J. Nitride Semicond. Res. 3, 8 (1998).

13. T. Detchprohm, S. Sano, S. Mochizuki, S. Kamiyama, H. Amano, and I. Akasaki, Phys. Stat. Sol.(a). 188, 799 (2001).

14. B. Beaumont, S. Haffouz, and P. Gibart, Appl. Phys. Lett. 72, 921 (1998). 